\title{
Capacidade para o trabalho e qualidade de vida de trabalhadores industriais
}

\author{
Work ability and quality of life of Brazilian industrial workers
}

Carolina Souza Neves da Costa ${ }^{1}$

Elizabeth Garcia de Freitas ${ }^{1}$

Lorena Cristina de Souza Mendonça ${ }^{1}$

Michele Elizabeth Rubio Alem ${ }^{1}$

Helenice Jane Cote Gil Coury ${ }^{1}$

${ }^{1}$ Departamento de Fisioterapia e Terapia Ocupacional, Centro de

Ciências Biológicas e da

Saúde, Universidade Federal

de São Carlos. Via

Washington Luis Km 235

Monjolinho. 13565-905

São Carlos SP.

costa.csn@gmail.com

\begin{abstract}
The scope of this study was to evaluate the quality of life and work ability of industrial workers by means of self-perception questionnaires. 100 industrial production line workers on the night shift participated in this study. Authorized Brazilian translations of the Work Ability Index (WAI) and the Abbreviated World Health Organization Quality of Life (WHOQOLBref) Assessment Instrument were applied. The results show an association between work ability and the whole set of domains of quality of life, presenting a closer association with the physical domain $(r=0.61)$. Furthermore, young men obtained higher ability to work results, while women aged between 30 and 39 had the lowest quality of life indices ( $p=0.027)$, especially in the Social Relationships and Environment domains. This shows that preventive guidelines for the improvement of aspects linked to the Physical domain are necessary both in the workplace and outside the workplace, since this interaction exacerbates the effects on the physical aspect in both spheres.
\end{abstract}

Key words Occupational groups, Self image, Questionnaires, World Health Organization, Workload, Health behavior
Resumo O objetivo do presente estudo foi avaliar, comparativamente, a qualidade de vida e a capacidade de trabalho de trabalhadores industriais, por meio dos indices de capacidade para o trabatho e de qualidade de vida. Participaram do estudo 100 trabalhadores de um setor noturno de linha de produção. Foram aplicadas traduções autorizadas do Indice de Capacidade para o Trabalho e o Instrumento Abreviado de Qualidade de Vida da Organização Mundial de Saúde. Os resultados evidenciam associação entre a capacidade para o trabalho e todo o conjunto dos domínios de qualidade de vida, apresentando melhor associação com o domínio Físico $(r=0,61)$. Além disso, os homens jovens obtiveram valores maiores para Capacidade de Trabalho ( $p=0,016)$, enquanto que as mulheres com idade entre 30-39 anos apresentaram os piores indices de qualidade de vida $(p=0,027)$, especialmente para os dominios Relações Sociais e Ambiente. Reforça-se, assim, a necessidade de diretrizes preventivas específicas para o aprimoramento dos aspectos ligados ao domínio Físico tanto no ambiente de trabalho como fora dele, uma vez que essa interação potencializa os efeitos no aspecto físico em ambas as esferas.

Palavras-chave Categorias de trabalhadores, Autoimagem, Organização Mundial da Saúde, Carga de trabalho, Conduta de saúde 


\section{Introdução}

Saúde é definida pela Organização Mundial de Saúde (OMS) não somente como ausência de doenças, mas também como um estado de bemestar físico, intelectual e social, o qual inclui aspectos relacionados à capacidade para o trabalho em sua concepção ${ }^{1}$. Dentro desse conceito mais abrangente, a utilização de instrumentos subjetivos que incluem a auto percepção e a autoavaliação de todos os fatores envolvidos é considerada uma opção rápida e eficaz para a investigação do bem-estar ${ }^{2}$. Dentre esses instrumentos, os questionários Índice de Capacidade para o Trabalho $(\mathrm{ICT})^{3}$ e o Instrumento de Avaliação Abreviado de Qualidade de Vida (WHOQOL-Breve) ${ }^{1}$ têm sido utilizados para avaliar, respectivamente, a capacidade para o trabalho e a qualidade de vida, porém em contextos independentes ${ }^{4-7}$.

O ICT foi originalmente desenvolvido para avaliar perdas na capacidade para o trabalho no envelhecimento. Ilmarinem e Tuomi ${ }^{4}$ afirmam que esta é um construto complexo, o qual é influenciado por fatores pessoais e específicos do trabalho. Esse indicador tem sido utilizado em diferentes contextos ocupacionais, e contribuído tanto na compreensão de perdas funcionais, como em seus possíveis mecanismos de compensação. Desta forma, Ilmarinem et al. ${ }^{8}$ verificaram o impacto da idade, a sobrecarga ocupacional e as mudanças de hábito de vida na capacidade para o trabalho em funcionários municipais finlandeses. Enquanto Nygard et al. ${ }^{9}$ acompanharam os efeitos do treinamento e da capacitação em uma linha de produção industrial alimentícia. No Brasil, Belusci e Fischer ${ }^{10}$ avaliaram as condições de trabalho, o envelhecimento e a capacidade funcional de servidores públicos e Walsh et al. ${ }^{5}$ avaliaram o impacto de fatores pessoais, clínicos e ocupacionais em trabalhadores industriais de uma empresa de médio porte.

O WHOQOL-Breve ${ }^{1}$ tem sido reconhecido como um importante instrumento de avaliação da qualidade de vida em diversas populações mundiais, incluindo trabalhadores de distintas ocupações. Dentre as diferentes populações avaliadas, Cimete et al. ${ }^{11}$ verificaram associação positiva entre qualidade de vida e satisfação no trabalho em enfermeiros turcos. Edimansyah et al. ${ }^{7}$ investigaram a relação entre fatores psicossociais relacionados ao trabalho e o índice de WHOQOL-Breve ${ }^{1} \mathrm{em}$ trabalhadores de uma indústria automobilística na Malásia. No Brasil, Oliveira Filho et al. ${ }^{12}$ avaliaram a percepção de qualidade de vida em médicos residentes e o efeitos desta no treinamento desses profissionais. Nunes e Freire ${ }^{13}$ e Penteado e Pereira ${ }^{14}$ avaliaram, respectivamente, a percepção da qualidade de vida em dentistas e em professores. Moreno et al. ${ }^{6}$ avaliaram funcionários técnico-administrativos, porém com o objetivo de verificar as propriedades psicométricas do instrumento. Entretanto, não foram localizados estudos que caracterizem a qualidade de vida em trabalhadores brasileiros no contexto industrial. Igualmente, não foram detectadas pesquisas na literatura internacional avaliando, concomitantemente, aspectos de capacidade para o trabalho e qualidade de vida em trabalhadores industriais. De fato, poucos estudos foram encontrados avaliando esses dois indicadores, especificadamente encontramos em enfermeiros ${ }^{15,16}$. No entanto, a avaliação associada desses dois indicadores pode trazer maior compreensão sobre fatores do trabalho (ICT) ${ }^{3} \mathrm{e}$ aspectos mais abrangentes de vida (WHOQOL) ${ }^{1}$ relacionados às perdas percebidas. Ainda, o detalhamento dessa análise por gênero e faixas etárias pode contribuir na identificação de aspectos mais críticos e grupos mais vulneráveis, o que por sua vez, pode auxiliar na determinação de medidas de controle mais eficazes. Assim, este estudo teve como objetivo avaliar, comparativamente, a qualidade de vida e a capacidade de trabalho em trabalhadores industriais de uma empresa brasileira de médio porte, por meio dos índices de WHOQOL-Breve ${ }^{1}$ e ICT ${ }^{3}$.

\section{Métodos}

\section{Sujeitos, atividades realizadas e cuidados éticos}

Participaram do estudo 100 indivíduos, sendo 34 homens e 66 mulheres com idade média de 34,8 ( $\pm 8,28$ anos). Esses indivíduos eram trabalhadores noturnos de um mesmo setor produtivo de uma empresa de médio porte, situada no interior do estado de São Paulo, produtora de materiais escolares e para escritório (lápis, lapiseiras, canetas, borrachas e apontadores). $\mathrm{O}$ tempo médio de trabalho na empresa era de 9,5 anos ( \pm 6,56 anos). O setor contava com 115 funcionários no momento da coleta, no entanto 3 se recusaram a participar e 12 encontravam-se em férias ou em banco de horas, assim a taxa de participação foi $87 \%$.

Esses indivíduos realizam atividades relacionadas ao processamento de madeira, pintura, carimbo e escolha de produtos. Para executar 
essas tarefas, trabalhavam nas posturas sentada e em pé e realizavam movimentos repetitivos de extremidades de membros superiores, contração muscular estática em músculos de cintura escapular, membros inferiores e coluna vertebral.

O estudo atendeu aos critérios éticos, tendo sido aprovado pelo Comitê de Ética local. Garantiu-se, dentre outros aspectos, o caráter voluntário do participante no estudo através da assinatura de um Termo de Consentimento Livre e Esclarecido.

\section{Materiais}

Foram utilizados o questionário para avaliação do Índice de Capacidade de Trabalho (ICT), desenvolvido pelo Instituto de Saúde Ocupacional de Helsinki, Finlândia (versão em português) ${ }^{3}$ e o questionário para a caracterização da qualidade de vida, desenvolvido pelo Grupo de Qualidade de Vida da Organização Mundial de Saúde (OMS) e denominado Instrumento Abreviado de Qualidade de Vida (WHOQOL- Breve) ${ }^{1}$.

O ICT avalia a capacidade de trabalho do indivíduo, por meio de questões objetivas (2) e subjetivas (5), as quais representam aspectos de sete diferentes esferas da vida dos trabalhadores, a saber: percepção da capacidade para o trabalho, exigências físicas e mentais do trabalho, doenças diagnosticadas, incapacidade para o trabalho, absenteísmo, prognóstico próprio e recursos mentais. O escore final do ICT pode variar entre 7 e 49 pontos, sendo classificado como: pobre (727 pontos), moderado (28-36 pontos), bom (3743 pontos) ou excelente (44-49 pontos).

O WHOQOL-Breve é constituído de 26 questões agrupadas em 4 domínios a saber: físico, psicológico, relações sociais e meio ambiente ${ }^{1}$.

\section{Procedimentos}

A coleta dos dados foi realizada em horário regular de trabalho, sem qualquer ônus para o trabalhador em forma de salário ou acréscimo de horas. Os questionários foram respondidos em uma sala próxima ao setor, em grupos de 8 a 10 pessoas. Inicialmente, era realizada uma leitura das questões por uma das pesquisadoras, que aguardava que cada questão fosse respondida individualmente. Dúvidas eventuais eram esclarecidas. O tempo gasto para o preenchimento dos questionários foi em média foi de 25 minutos. Para a manutenção do sigilo do trabalhador, os questionários foram codificados de maneira adequada.

\section{Análise dos Dados}

O cálculo dos escores dos dois questionários foi efetuado conforme procedimentos usuais desses instrumentos ${ }^{3,17}$. Na análise dos resultados de qualidade de vida foi calculada a média ponderada para os 4 domínios, representando o escore geral de qualidade de vida (QV média) ${ }^{14}$. Valores de normalidade dos dados foram determinados pelo teste de Shapiro-Wilks. Como os dados referentes ao ICT não seguiram os parâmetros de distribuição normal, estes foram ajustados $\left(\mathrm{ICT}^{*}=\mathrm{ICT}^{4,48}\right)$, possibilitando a estatística paramétrica.

Para a verificação de diferenças nos índices gerais de qualidade de vida, capacidade para o trabalho, e seus respectivos domínios e esferas entre grupos classificados por faixa etária e gênero foi utilizada análise de variância (ANOVA) por dois fatores. Quando verificada a influência significante para algum dos fatores, foi realizado o teste post hoc de Tukey para a localização das diferenças. Correlações de Pearson foram aplicadas para verificar associações entre os índices de ICT e os domínios advindos do WHOQOL-Breve.

\section{Resultados}

Um valor médio de 40,27 (escala de 7-49) foi identificado para a capacidade para o trabalho no grupo estudado. Quanto à classificação do ICT, os indivíduos se distribuíram da seguinte maneira: 3\% encontravam-se na categoria Pobre, 15\% em Moderado, 52\% Bom e 30\% na categoria Excelente. Os escores identificados nas diferentes esferas do ICT foram: 8,55 (0-10) em capacidade para o trabalho, 7,8 (0-10) em exigências físicas e mentais, 4,88 (0-7) em doenças diagnosticadas, 5,33 (0-6) na incapacidade para o trabalho, 4,29 (0-5) em absenteísmo, 6,16 (0-7) em prognóstico próprio e 3,43 (0-4) para recursos mentais.

Um valor médio de 66,52 foi identificado para os escores de QV em uma escala de 0-100. Os escores de WHOQOL-Breve identificados nos respectivos domínios foram: 69,40 para o Físico, 68,91 para o Psicológico, 71,96 para Relações Sociais e 55,79 para Ambiente.

A Figura 1 apresenta valores médios, medianas e distribuição dos resultados distribuídos por gênero e idade. Quando os grupos de ICT foram comparados entre si, uma diferença significativa ocorreu para o grupo mais jovem masculino (2029 anos), que apresentou níveis maiores de capacidade para o trabalho $(\mathrm{p}=0,016)$. Já para os 
índices de QV, o grupo feminino de 30-39 anos apresentou índices significativamente menores que os demais $(\mathrm{p}=0,027)$.
A Tabela 1 apresenta as médias e os desvios-padrão dos valores para as diferentes esferas de ICT de acordo com o gênero e a idade.

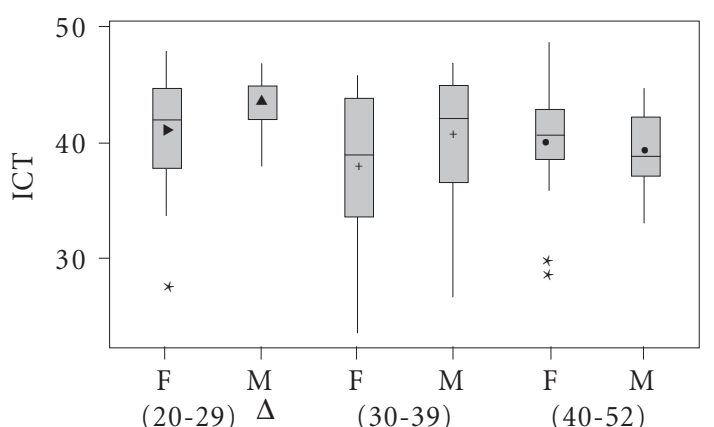

Categorias Idade $\Delta(\mathrm{p}<0.05)$

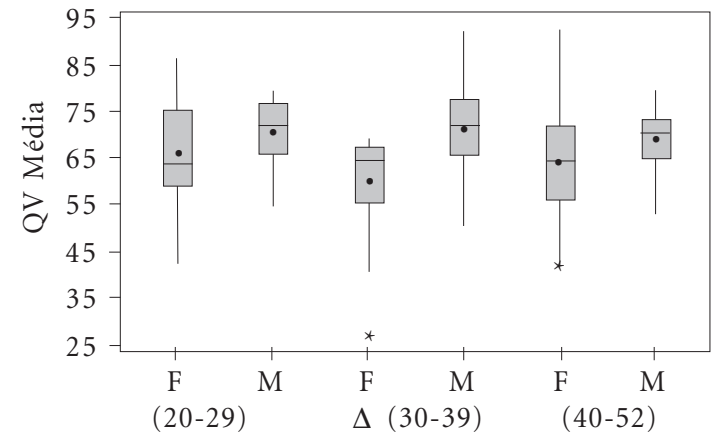

Categorias Idade $\Delta(\mathrm{p}<0.05)$

Figura 1. Valores médios, medianas e distribuição dos escores de ICT e QV para homens e mulheres por faixas etárias. Diferenças significativas entre os grupos são mostradas pelo símbolo $\Delta$. ( $\mathrm{F}=$ feminino; $\mathrm{M}=$ masculino)

Tabela 1. Valores médios e desvio padrão dos escores das esferas de ICT para os diferentes gêneros e faixas etárias. Diferença significativa entre os grupos são mostradas pelo símbolo *.

\begin{tabular}{|c|c|c|c|c|c|c|c|c|}
\hline Esferas de ICT & Gênero & Média & $\mathrm{DP}$ & $p$ & Idade & Média & DP & $p$ \\
\hline \multirow{3}{*}{$\begin{array}{l}\text { Capacidade atual } \\
\text { para o trabalho }\end{array}$} & $\mathrm{F}$ & 8,53 & 1,20 & & $20-29$ & 8,81 & 1,17 & \\
\hline & & & & & $30-39$ & 8,30 & 1,15 & \\
\hline & M & 8,58 & 1,20 & 0,82 & $40-52$ & 8,54 & 1,59 & 0,245 \\
\hline \multirow{3}{*}{$\begin{array}{l}\text { Exigências Físicas } \\
\text { e Mentais }\end{array}$} & $\mathrm{F}$ & 7,69 & 1,37 & & $20-29$ & 8,00 & 0.95 & \\
\hline & & & & & $30-39$ & 7,57 & 1,30 & \\
\hline & M & 8,00 & 1,04 & 0,26 & $40-52$ & 7,82 & 1,48 & 0,40 \\
\hline \multirow[t]{3}{*}{ Doenças Diagnosticadas } & $\mathrm{F}$ & 4,56 & 2,16 & & $20-29$ & 8,81 & 1,17 & \\
\hline & & & & & $30-39$ & 8,30 & 1,15 & \\
\hline & M & 5,50 & 1,67 & $0,02^{\star}$ & $40-52$ & 7,82 & 1,48 & 0,51 \\
\hline \multicolumn{9}{|l|}{ Incapacidade } \\
\hline \multirow[t]{3}{*}{ para o trabalho } & $\mathrm{F}$ & 5,22 & 0,79 & & $20-29$ & 5,40 & 0,71 & \\
\hline & & & & & $30-39$ & 5,21 & 0,85 & \\
\hline & M & 5,52 & 0,70 & 0,06 & $40-52$ & 4,65 & 1,98 & 0,56 \\
\hline \multirow[t]{3}{*}{ Absenteísmo } & $\mathrm{F}$ & 4,30 & 0,82 & & $20-29$ & 4,53 & 0,92 & \\
\hline & & & & & $30-39$ & 4,15 & 1,00 & \\
\hline & M & 4,26 & 1,08 & 0,60 & $40-52$ & 4,20 & 1,02 & 0,21 \\
\hline \multirow[t]{3}{*}{ Prognóstico Próprio } & $\mathrm{F}$ & 6,18 & 1,62 & & $20-29$ & 6,81 & 0,73 & \\
\hline & & & & & $30-39$ & 5,45 & 2,01 & \\
\hline & M & 6,11 & 1,73 & 0,90 & $40-52$ & 6,14 & 1,71 & $0,00^{*}$ \\
\hline \multirow[t]{3}{*}{ Recursos Mentais } & $\mathrm{F}$ & 3,42 & 0,60 & & $20-29$ & 3,40 & 0,55 & \\
\hline & & & & & $30-39$ & 3,39 & 0,65 & \\
\hline & M & 3,44 & 0,61 & 0,88 & $40-52$ & 3,48 & 0,61 & 0,79 \\
\hline
\end{tabular}

$\mathrm{F}=$ Feminino $; \mathrm{M}=$ Masculino $; \mathrm{DP}=$ Desvio Padrão 
Quando os grupos foram comparados entre si dentro de cada esfera do ICT, o grupo de trabalhadores do sexo masculino mostrou valores significativamente maiores na de doenças diagnosticadas quando comparado ao grupo feminino. Os resultados de incapacidade para o trabalho foram maiores para os homens, atingindo valores limítrofes de significância. Os indivíduos entre 20-29 anos de idade apresentaram médias significativamente superiores para o prognóstico próprio quanto à capacidade para a realização da mesma tarefa daqui a dois anos do que os demais grupos. A Tabela 2 apresenta as médias e os desvios-padrão para os domínios de WHOQOL-Breve por gênero e faixas etárias. Somente os domínios Relações Sociais e Ambiente mostraram médias significativamente menores paras as mulheres.

Dentre os domínios de qualidade de vida, o ICT mostrou correlação significativa mais forte no Físico $(\mathrm{r}=0,61)$, seguido pelos Psicológico $(\mathrm{r}$ $=0,44)$, Relações Sociais $(r=0,42)$ e Ambiente $(0,32)$. Todas as correlações descritas acima foram significativas em nível de $\mathrm{p}<0,01$.

\section{Discussão}

O ICT foi desenvolvido para avaliar a percepção individual de capacidade para o trabalho relacionado às exigências deste, ao estado de saúde (baseado no número de doenças diagnosticadas e absenteísmo), aos recursos mentais e à interação do indivíduo no ambiente laboral ${ }^{4}$, sendo um instrumento dirigido para este espaço. Por outro lado, o WHOQOL-Breve ${ }^{1}$, considerado como um dos questionários mais abrangentes, avalia as várias dimensões da qualidade de vida ${ }^{17}$. No entanto, os resultados relatados aqui indicam que a capacidade para o trabalho na população industrial estudada está significativamente correlacionada com a percepção de todos os domínios da qualidade de vida. Isso sugere que a percepção da capacidade para o trabalho individual não somente está associada aos fatores relacionados ao trabalho, mas poderia expressar, pelo menos em parte, a percepção da qualidade de vida fora do ambiente laboral.

Nos estudos encontrados, associando a qualidade de vida e a capacidade para o trabalho por meio dos instrumentos WHOQOL-Breve e ICT, Chiu et al. ${ }^{15}$ e Milosevic et al. ${ }^{16}$ avaliaram enfermeiras de diferentes departamentos e centros médicos em Taiwan e na Croácia, respectivamente, e verificaram que Ambiente e Físico eram os domínios de qualidade de vida que mais correlacionaram-se com a capacidade de trabalho percebida, ressaltando as condições de risco à segurança e à exigência de revezamento de turnos e de esforço físico, no contexto específico que essas mulheres trabalhavam. No presente estudo, o domínio Físico do instrumento WHOQOL-breve foi o que mais fortemente correlacionou-se com o ICT, sugerindo que a percepção de uma boa capacidade física parece expressar-se também em uma boa capacidade para o trabalho no contexto industrial. Assim, tal achado corrobora os resultados da revisão sistemática de Van den Berg et al. ${ }^{18}$

Tabela 2. Valores médios e desvio padrão dos domínios de QV para os diferentes gêneros e faixas etárias.

\begin{tabular}{|c|c|c|c|c|c|c|c|c|}
\hline Domínios de QV & Gênero & Média & DP & $p$ & Idade & Média & DP & $p$ \\
\hline \multirow[t]{3}{*}{ Físico } & $\mathrm{F}$ & 67,74 & 17,16 & & $20-29$ & 73,00 & 17,04 & \\
\hline & & & & & $30-39$ & 65,48 & 15,34 & \\
\hline & M & 72,62 & 12,44 & 0,14 & $40-52$ & 69,80 & 14,68 & 0,15 \\
\hline \multirow[t]{3}{*}{ Psicológico } & $\mathrm{F}$ & 67,45 & 15,83 & & $20-29$ & 71,19 & 12,74 & \\
\hline & & & & & $30-39$ & 67,73 & 17,31 & \\
\hline & M & 71,74 & 12,44 & 0,14 & $40-52$ & 67,94 & 13,08 & 0,56 \\
\hline \multirow[t]{3}{*}{ Relações Sociais } & $\mathrm{F}$ & 69,71 & 17,33 & & $20-29$ & 72,22 & 12,04 & \\
\hline & & & & & $30-39$ & 74,85 & 17,89 & \\
\hline & M & 76,32 & 12,96 & $0,03^{*}$ & $40-52$ & 69,00 & 17,76 & 0,33 \\
\hline \multirow[t]{3}{*}{ Ambiente } & $\mathrm{F}$ & 54,47 & 11,72 & & $20-29$ & 54,00 & 12,18 & \\
\hline & & & & & $30-39$ & 55,24 & 10,56 & \\
\hline & M & 58,35 & 9,47 & $0,05^{\star}$ & $40-52$ & 57,94 & 10,55 & 0,33 \\
\hline
\end{tabular}

$\mathrm{F}=$ Feminino $\mathrm{M}=$ Masculino $\mathrm{DP}=$ Desvio Padrão 
sobre o efeitos de fatores relacionados ao trabalho e ao indivíduo no índice de capacidade para o trabalho, a qual afirma que os fatores relacionados à satisfação na vida e à capacidade para o trabalho parecem possuir uma relação interativa e mútua, sendo determinados conjuntamente.

De acordo com Jansen et al. ${ }^{19}$, interações entre a vida profissional e familiar são importantes na percepção da capacidade para o trabalho e o bem-estar emocional. Enquanto que Hildebrandt e Bongers ${ }^{20}$ verificaram efeitos positivos de atividades físicas e de lazer na percepção da capacidade para o trabalho físico e social. Infere-se assim que o trabalho e suas dimensões podem ser expressados por fatores ocupacionais e nãoocupacionais. Desta maneira, programas de promoção de saúde do trabalhador que tenham como foco o indivíduo e todas as suas facetas são essenciais, confirmando a necessidade do desenvolvimento de um novo conceito de saúde do trabalhador para além do ambiente laboral.

No que tange ao desempenho da população estudada para cada instrumento, verificou-se que $82 \%$ dos trabalhadores foram classificados como tendo boa e excelente capacidade para o trabalho, com uma média de 40,27 para o ICT. À luz de outros estudos de populações laborais, constatam-se semelhanças entre a população estudada e a da Finlândia, a qual apresentou $85 \%$ de trabalhadores de uma indústria de porte médio nas categorias bom/excelente ${ }^{3}$. Porém, os nossos resultados são piores do que os apresentados por trabalhadores de setores administrativos no Brasil $^{10}$, e possuem escores mais baixos do que o estrato de trabalhadores que apresentam os piores escores de ICT de indústrias polonesas ${ }^{21}$.

Quando se analisa os elementos relacionados à qualidade de vida da população, observam-se também médias mais baixas para QV média e para domínios Físico e Psicológico em comparação com a pontuação da população industrial malasiana ${ }^{7}$ e de setores administrativos brasileiros ${ }^{6}$. Médias mais baixas para todos os domínios do WHOQOL-breve foram obtidos pela nossa população em comparação com o grupo de brasileiros estudados pela Organização Mundial de Saúde ${ }^{17}$, e ainda piores do que aqueles apresentados por prestadores de serviços especializados brasileiros, como dentistas ${ }^{13} \mathrm{e}$ médicos residentes ${ }^{12}$. Tais achados indicam, assim, a possibilidade real de que as condições de trabalho na indústria brasileira sejam piores do que as existentes em outros países e em outros setores laborais brasileiros.

O detalhamento da análise por gênero e faixas etárias identificou que o grupo mais jovem masculino apresentou melhor capacidade para o trabalho do que os demais. Segundo Sluiter et al. ${ }^{22}$, a idade é fator determinante no declínio de funções fisiológicas como força física, capacidade respiratória, hormonais e mentais que estão intimamente ligadas à perda da capacidade funcional para as atividades que requerem maior exigência física. Além disso, Kujala et al. ${ }^{23}$ argumenta que trabalhadores jovens, principalmente homens, possuem menor insegurança no ambiente laboral, maior otimismo quanto à percepção de capacidade para o trabalho. Tais fatores parecem explicar os melhores índices de capacidade para o trabalho e melhor disponibilidade física e otimismo em relação ao futuro para estes, o que corrobora também os resultados aqui apresentados sobre a percepção do prognóstico próprio em realizar a mesma tarefa daqui a 2 anos.

Os resultados revelaram, ainda, que as mulheres com idade entre 30-39 anos apresentaram índices de QV significativamente menores do que os homens, principalmente quanto aos domínios Relações Sociais e Ambiente. Fatores relacionados à qualidade de vida feminina são usualmente interpretados à luz das esferas simultâneas de atuação da mulher como operária, esposa, mãe e dona de casa. Desta forma, a atividade não-ocupacional, como o trabalho doméstico e os cuidados com filhos, cria jornadas extras de trabalho ${ }^{24}$. Tais fatores podem ter contribuído para explicar menores índices femininos na percepção da qualidade de vida.

Outro fator importante a ser considerado aqui e que pode estar relacionado ao anterior, é o fato de que os trabalhadores avaliados desempenharam suas atividades no período noturno. Kaliterna et al..$^{25}$,em um estudo analisando qualidade de vida em diferentes horários de trabalho, concluíram que mulheres assistentes sociais com menos de 40 anos de idade que desempenham jornada noturna apresentam menores índices de qualidade de vida, sentem-se mais infelizes do que grupos com idade mais avançada. Trabalhar em turnos noturnos provoca dificuldades na vida familiar e restrições à vida social e às atividades de lazer com companheiro, amigos e filhos ${ }^{26}$. Esses aspectos podem explicar, pelo menos em parte, os menores índices registrados para os elementos do domínio Ambiente, a saber: percepção de indisponibilidade para o lazer, insegurança financeira, os quais podem ser expressos igualmente no déficit de especificidades advindas do domínio Relações Sociais, como manutenção de laços sociais e familiares.

O desenho transversal deste estudo não possibilita concluir sobre causalidade entre os fatores. $\mathrm{O}$ 
planejamento do estudo também não avaliou trabalhadores ausentes do trabalho por razões médicas, incluindo aqueles afetados por lesões relacionadas ao trabalho. Assim, os resultados do estudo são aplicáveis apenas aos trabalhadores do setor industrial que estavam ativos durante a avaliação.

Além disso, características sociodemográficas e econômicas e a existência de um ambiente de trabalho menos favorável, apresentando com alta demanda física e ritmo de trabalho repetitivo e intenso para a população estudada, além de características individuais, como obesidade, são aspectos que devem ser considerados para explicar os resultados descritos aqui. Embora as atividades tenham sido muito similares entre os indivíduos, não influenciando na variabilidade reportada, não foi realizada uma análise ergonômica da atividade, o que pode ser considerado como uma limitação do presente estudo.

Em conclusão, os resultados indicam que a capacidade para o trabalho foi significativamente associada com a percepção de qualidade de vida nos trabalhadores industriais estudados e, essa associação foi mais forte para os aspectos físicos da avaliação. Além disso, o grupo masculino mais jovem apresentou maior capacidade funcional, enquanto que mulheres de faixa etária mediana (3039 anos) apresentaram os piores índices de qualidade de vida. Dessa forma, futuros estudos devem ser efetuados com populações industriais brasileiras objetivando conhecer os fatores específicos associados às facetas de pior desempenho para cada um dos índices avaliados. Os resultados aqui presentes, ainda, reforçam a necessidade de diretrizes preventivas específicas para o aprimoramento dos aspectos ligados ao domínio Físico tanto no ambiente de trabalho, tais como intervenções ergonômicas, como fora dele, uma vez que essa interação potencializa os efeitos sobre aspectos físicos em ambas as esferas.

\section{Colaboradores}

CSN Da Costa contribui com a escrita, planejamento experimental, coleta de dados e discussão dos resultados. EG Freitas e LCS Mendonça contribuíram com o planejamento experimental, a coleta de dados e a discussão dos dados. MER Alem contribui com o planejamento, a coleta de dados e a coorientação. HJCG Coury orientou o trabalho.

\section{Agradecimentos}

Agradecemos à Cristina Nardim pela valiosa contribuição com a análise estatística utilizada nesse estudo. Agradecemos a todos os trabalhadores voluntários envolvidos neste estudo. 


\section{Referências}

1. The WHOQOL Group. Development of the world Health organization WHOQOL-Bref quality of life assessment. Psychol Med 2000; 28(3):551-558.

2. Bowling A. Mode of questionnaire administration can have serious effects on data quality. J Public Health (Oxf) 2005; 27(3):281-291.

3. Tuomi K, Ilmarienn J, Katajarine L, Tulkki A. Índice de capacidade para o trabalho. Tradução de FM Fischer. Helsinki: Instituto Finlandês de Saúde Ocupacional; 1997.

4. Ilmarinem J, Tuomi K. Past present and future of work ability. People Work Res Rep 2004; 65:1-25.

5. Walsh IAP, Corral S, Franco RN, Canetti EEF, Alem MER, Coury HJCG. Capacidade para o trabalho em indivíduos com lesões músculo-esqueléticas crônicas. Rev Saude Publica 2004; 38(2):149-156.

6. Moreno A, Faerstein E, Werneck G, Lopes CS, Chor D. Propriedades psicométricas do Instrumento Abreviado de Avaliação de Qualidade de Vida da Organização Mundial da Saúde no estudo pró-saúde. Cad Saude Publica 2006; 22(12):2585-2597.

7. Edimansyah B, Rusli BN, Naing L, Mohamed Rusli BA, Winn T. Relationship of psychosocial work factors and health-related quality of life in male automotive assembly workers in Malaysia. Industrial Health 2007; 45:437-448.

8. Ilmarinen J, Tuomi K, Seitsamo J. New dimensions of work ability. Intern Congress Series 2005; 1280:3-7.

9. Nygard CH, Manka ML, Huhtala H. Work ability, perceived exertion and training during production changes in food factory. Adv Occup Ergon Safe 1996; 21(2):931-935.

10. Bellusci S, Fischer F. Envelhecimento funcional e condições de trabalho em servidores forenses. Rev Saude Publica 1999; 33(6):602-609.

11. Cimete G, Gencalp N, Keskin G. Quality of life and job satisfaction of nurses. J Nurs Care Qual 2003; 18(2):151-158.

12. Oliveira Filho GR, Sturm EJ, Sartorato AE. Compliance with common program requirements in Brazil: its effects on resident's perceptions about quality of life and the educational environment. Acad Med 2005; 80(1):98-102.

13. Nunes MF, Freire MCM. Qualidade de vida de cirurgiões-dentistas que atuam em um serviço público. Rev Saude Publica 2006; 40(6):1019-1026.

14. Penteado RZ, Pereira IM. Quality of life and vocal health of teachers. Rev Saude Publica 2007; 41(2):236243.

15. Chiu M, Wang MJ, Lu C, Pan S, Kumashiro M, Ilmarienm J. Evaluating work ability and quality of life for clinical nurses in Taiwan. Nurs Out 2007; 6(55):318-326.
16. Milosevic M, Golubic R, Knezevic B, Golubic K, Bubas M, Mustajbegovic J. Work ability as a major determinant of clinical nurses' quality of life. J Clin Nurs 2011; 20(19-20):2931-2938.

17. Skevington S, Lotfy M, O’Connell KA, WHOQOL Group. The World Health Organization's WHOQOL-Bref quality of life assessment: Psychometric properties and results of the international field trial. A report from WHOQOL Group. Qual Life Res 2004; 13(2):299-310.

18. Van den Berg TI, Elders LA, de Zwart BC, Burdof A. The effects of work-related and individual factors on the Work Ability Index: a systematic review. Occup Environ Med 2009; 66(4):211-220.

19. Jansen NWH, Kant IJ, van Amelsvoort LG, Kristensen TS, Swaen GM, Nijhuis FJ. Work-family conflict as risk factor for sickness absence. Occup Environ Med 2006; 63(7):488-494.

20. Hildebrandt V, Bongers P. The relationship between leisure time, physical, activities and musculoskeletal symptoms and disability in worker populations. Intern Arch Occup Health 2000; 73(8):507-518.

21. Bugasjska J, Lastowiecka E. Life style, work environment factors and work ability in different occupations. Intern Congress Series 2005; 1280:247-252.

22. Sluiter JK, High-demand jobs: age-related diversity in work ability? Appl Ergon 2006; 37(4):429-440.

23. Kujala V, Remes J, Tammelin T, Laitinen J. Classification of Work Ability Index among Young emplyees. Occup Med (Lond) 2005; 55(5):399-401.

24. Johansson G, Huang Q, Lindfors P. A life-span perspective on women's careers, health, and well-being. Social Scien \& Med 2007; 65(4):685-697.

25. Kaliterna L, Prismic L P, Zganec N. Quality of life, life satisfaction and happiness in shift-and non-shiftworkers. Rev Saude Publica 2004; 38(Supl.):3-10.

26. Tepas D, Bares-Farrel JL, Bobko N, Fischer F, Golec II, Kaliterna L. The impact of night work on subjective reports of well-being: an exploratory study of health care workers from five nations. Rev Saude Publica 2004; 38(Supl.):26-31.

Artigo apresentado em 01/03/2012

Aprovado em 09/03/2012

Versão final apresentada em 22/03/2012 